\title{
Energy distribution of relativistic electrons in the young supernova remnant G1.9+0.3
}

\author{
Xiao-na Sun ${ }^{a}$, Felix Aharonian $^{a b c}$, and Rui-zhi Yang ${ }^{* a}$ \\ ${ }^{a}$ Max-Planck-Institut für Kernphysik, P.O. Box 103980, 69029 Heidelberg, Germany \\ ${ }^{b}$ Dublin Institute for Advanced Studies, 31 Fitzwilliam Place, Dublin 2, Ireland \\ ${ }^{c}$ MEPHI, Kashirskoe shosse 31, 115409 Moscow, Russia. \\ E-mail: sun@mpi-hd.mpg •de, ryang@mpi-hd.mpg.de, \\ Felix.Aharonian@mpi-hd.mpg.de
}

\begin{abstract}
The broad-band X-ray observations of the youngest known galactic supernova remnant (SNR), G1.9+0.3, provide unique information about the particle acceleration at the early stages of evolution of supernova remnants. Based on the publicly available X-ray data obtained with the Chandra and NuSTAR satellites over two decades in energy, we derived the energy distribution of the relativistic electrons under the assumption that detected X-rays are of entirely synchrotron origin. The acceleration of electrons was found to be an order of magnitude slower than the maximum rate provided by the shock acceleration in the nominal Bohm diffusion regime. We discuss the implications of this result in the context of contribution of SNRs to the Galactic Cosmic Rays at PeV energies.
\end{abstract}

35th International Cosmic Ray Conference - ICRC2017

10-20 July, 2017

Bexco, Busan, Korea

\footnotetext{
* Speaker.
} 


\section{Introduction}

Supernova Remnants (SNRs) are believed to be the sites where the bulk of Galactic cosmic rays (CRs) are accelerated up to $\mathrm{PeV}$ energies $\left(1 \mathrm{PeV}=10^{15} \mathrm{eV}\right)$ (see, e.g, [1, 2]). In recent years, significant progress has been achieved in a few directions of exploring the CR acceleration in SNRs, in particular using the $\gamma$-ray observations in the $\mathrm{MeV} / \mathrm{GeV}$ and $\mathrm{TeV}$ energy bands (see, e.g, [3]). In particular, the detection of the so-called $\pi^{0}$-decay bump in the spectra of several mid-age SNRs, is considered as a substantial evidence of acceleration of protons and nuclei in SNRs. Moreover, the detection of more than ten young (a few thousand years old or younger) SNRs in TeV $\gamma$-rays highlights these objects as efficient particle accelerators, although the very origin of $\gamma$-rays (leptonic or hadronic?) is not yet firmly established. More disappointingly, so far all $\mathrm{TeV}$ emitting SNRs do not show energy spectra that would continue as a hard power-law beyond $10 \mathrm{TeV}$. For a hadronic origin of detected $\gamma$-rays, the "early" cutoffs in the energy spectra of $\gamma$-rays around or below 10 $\mathrm{TeV}$ imply a lack of protons inside the shells of SNRs with energies significantly larger than 100 $\mathrm{TeV}$, and, consequently, SNRs do not operate as PeVatrons. However, there are two possibilities that would allow us to avoid such a dramatic conclusion for the current paradigm of Galactic CRs:

(i) The detected $\mathrm{TeV}$ gamma-rays are of leptonic (Inverse Compton) origin. Of course, alongside the relativistic electrons, protons and nuclei can (should) be accelerated as well, but we do not see the related $\gamma$-radiation because of their ineffective interactions caused by the low density of ambient gas;

(ii) SNRs do accelerate protons to PeV energies, however it occurs at early stages of evolution of SNRs when the shock speeds exceed $10,000 \mathrm{~km} / \mathrm{s}$; we do not see the corresponding radiation well above $10 \mathrm{TeV}$ because the $\mathrm{PeV}$ protons have already left the remnant.

Both these scenarios significantly limit the potential of gamma-ray observations for the search for CR PeVatrons. Fortunately, there is another radiation component, which contains independent and complementary information about these extreme accelerators. It is related to the synchrotron radiation of accelerated electrons, namely to the shape of the energy spectrum of radiation in the cutoff region which can serve as a distinct signature of the acceleration mechanism and its efficiency. In the shock acceleration scheme, the maximum energy of accelerated particles, $E_{0} \propto B v_{\mathrm{sh}}^{2}$. Therefore, the epoch of the first several hundred years of evolution of a SNR, when the shock speed $v_{\text {sh }}$ exceeds $10,000 \mathrm{~km} / \mathrm{s}$ and the magnetic field is large $(B \gg 10 \mu \mathrm{G}$ ) could be an adequate stage for operation of a SNR as a PeVatron, provided, of course, that the shock acceleration proceeds close to the Bohm diffusion limit (see, e.g, [4]). Remarkably, in this regime, the cutoff energy in the synchrotron radiation of the shock-accelerated electrons is determined by a single parameter, $v_{\mathrm{sh}}^{2}[5,6]$. Therefore, for the known shock speed, the position of the cutoff contains an unambiguous information about the acceleration efficiency. For $v_{\mathrm{sh}} \simeq 10,000 \mathrm{~km} / \mathrm{s}$, the synchrotron cutoff in the spectral energy distribution (SED) is expected around $10 \mathrm{keV}$. Thus, the study of synchrotron radiation in the hard X-ray band can shed light on the acceleration efficiency of electrons, and, consequently, provide an answer as to whether or not these objects can operate as CR PeVatrons, given that in the shock acceleration scheme the acceleration of electrons and protons is expected to be identical. In this regard, G1.9+0.3, the youngest known SNR in our Galaxy [7, 8], is a perfect object to explore this unique tool. 
The X-ray observations with the Chandra and NuSTAR satellites $[9,10]$ cover a rather broad energy interval which is crucial for the study of the spectral shape of synchrotron radiation, in particular in the cutoff region. Such a study has been conducted by the team of the NuSTAR collaboration [10].

In this paper we present the results of our own analysis of the NuSTAR and Chandra data with an emphasis on the study of the SED of X-ray radiation over two decades, from $0.3 \mathrm{keV}$ to $30 \mathrm{keV}$. Using the synchrotron emissivity of relativistic electrons and the Markov Chain Monte Carlo (MCMC) technique, we derive the energy distribution of electrons responsible for X-rays and discuss the astrophysical implications of the obtained results.

\section{X-ray observations}

The recent hard X-ray observations of G1.9+0.3 by the NuSTAR satellite are uniquely useful for understanding the acceleration and radiation processes of ultrarelativistic electrons in SNRs at the early stages of their evolution. Detailed study of the NuSTAR data, combined with the Chandra observations at lower energies, has been comprehensively carried out by [10]. In particular, it was found that the source can be resolved into two bright limbs with similar spectral features. The combined Chandra and NuSTAR datasets have been claimed to be best described by the so-called srcut model [11] or by the power-law function with an exponential cutoff. The characteristic cutoff energies in these two fits have been found around $3 \mathrm{keV}$ and $15 \mathrm{keV}$, respectively [10].

To further investigate the features of the X-ray spectrum in the cutoff region we performed an independent study based on the publicly available Chandra and NuSTAR X-ray data. For NuSTAR, we used the set of three observations with ID 40001015003, 40001015005, and 40001015007, including both the focal plane A (FPMA) and B (FPMB) modules. The data have been analysed using the HEASoft version 6.16, which includes NuSTARDAS, the NuSTAR Data Analysis Software package (the version 1.7.1 with the NuSTAR CALDB version 20150123). For the Chandra data, we used the ACIS observations with ID 12691, 12692 and 12694. The Chandra data reduction was performed using version 4.7 of the CIAO (Chandra Interactive Analysis of Observations) package.

The spectral shape of synchrotron radiation in the cutoff region is sensitive to the spectrum of highest energy electrons which, in turn, depends on the electron acceleration and energy loss rates. To explore a broad class of spectra, we describe the spectrum of X-rays in the following general form:

$$
\frac{\mathrm{d} N}{\mathrm{~d} \varepsilon}=A E^{-\Gamma} \exp \left[-\left(\varepsilon / \varepsilon_{0}\right)^{\beta}\right]
$$

The change of the index $\beta$ in the second (exponential) term allows a broad range of spectral behaviour in the cutoff region. For example, $\beta=0$ implies a pure power-law distribution, while $\beta=1$ corresponds to a power-law with a simple exponential cutoff.

In the fitting procedure, in addition to the three parameters $\varepsilon_{0}, \Gamma$, and $\beta$, one should introduce one more parameter, the column density $N_{\mathrm{H}}$, which takes into account the energy-dependent absorption of X-rays. We fix this parameter to the value found by [10] from the fit of data by their srcut spectral model . Strictly speaking, the best fit value of the column density should be different for different spectral models. To check the impact of different spectral models on the column 
density, we adopted different functions leaving the column density as a free parameter in the fitting procedure. We found that the difference of the best fit column density and the above fiducial value is less than several percent. Therefore, in order to keep the procedure simple and minimise the number of free parameters, we adopt the value $N_{\mathrm{H}}=7.23 \times 10^{22}$ from the paper of [10].

The results of our fit of the NuSTAR and Chandra spectral points using the model "powerlaw with exponential cutoff" in the general form of Eq.(1), that is, leaving $\beta, \Gamma$, and $\varepsilon$ as free parameters, are shown in Table 1. One can see that the best fit gives a rather narrow range of the index $\beta$ around $1 / 2$. In Table 1 we also separately show the results of the fits with three fixed values of $\beta: 0,1 / 2$, and 1 . While the pure-power-law spectrum $(\beta=0)$ can be unambiguously excluded, the model of power-law with a simple exponential cutoff $(\beta=1)$ is not favourable either. It is excluded at the $3 \sigma$ statistical significance level. In summary, the combined Chandra and NuSTAR data are best described by the index $\beta \approx 0.5$ and $\varepsilon_{0} \approx 1.5 \mathrm{keV}$.

Whereas $\beta=1 / 2$ seems to be a natural outcome (see below), the cutoff energy around 1.5 $\mathrm{keV}$ is a rather unexpected result. Namely, it implies that the acceleration of electrons in G1.9+0.3 proceeds significantly slower than one would anticipate given the very large, $14,000 \mathrm{~km} / \mathrm{s}$ shock speed [13]. This can be seen from the comparison of the SED of G1.9+0.3 with one of the most effective particle accelerators in our Galaxy, $\approx 1600$ year old SNR RX J1713.4-3946 (see left panel of Fig.1). The cutoff energy in the synchrotron spectrum of shock-accelerated electrons is proportional to the square of shock speed $v_{\mathrm{sh}}^{2}$ [5]. Therefore, in order to exclude the difference in the cutoff energies caused by the difference in the shock speeds, we rescale the energies of the spectral points of RX J1713.4-3946 by the factor $\left(v_{\mathrm{sh}} / 14,000 \mathrm{~km} / \mathrm{s}\right)^{2}$, where the shock speed of RX J1713.4-3946 is about $v_{\mathrm{sh}} \simeq 4,000 \mathrm{~km} / \mathrm{s}$ [14]. After such a normalisation, the cutoff energy of RX J1713.4-3946 becomes an order of magnitude higher than the cutoff in G1.9+0.3. The acceleration of electrons in RX J1713.4-3946 proceeds close to the Bohm diffusion limit thus providing an acceleration rate close to the maximum value $[14,15]$. Consequently, we may conclude that the current acceleration rate of electrons in G1.9+0.3 is lower, by an order of magnitude, compared to the maximum possible rate.

The maximum acceleration rate of particles is achieved when it proceeds in the Bohm diffusion limit. In the energy-loss-dominated regime, the spectra of synchrotron radiation can be expressed by simple analytical formulae [6]. Because of compression of the magnetic field, the overall synchrotron flux of the remnant is dominated by the radiation from the downstream region (see Fig.1). The SED of the latter can be presented in the following form [6]

$$
\varepsilon^{2} \frac{\mathrm{d} N}{\mathrm{~d} \varepsilon} \propto \varepsilon^{2}\left(\varepsilon / \varepsilon_{0}\right)^{-1}\left[1+0.38\left(\varepsilon / \varepsilon_{0}\right)^{0.5}\right]^{11 / 4} \exp \left[-\left(\varepsilon / \varepsilon_{0}\right)^{1 / 2}\right] .
$$

with

$$
\varepsilon_{0}=\hbar \omega_{0}=\frac{2.2 \mathrm{keV}}{\eta\left(1+\kappa^{1 / 2}\right)^{2}}\left(\frac{u_{1}}{3000 \mathrm{~km} \mathrm{~s}^{-1}}\right)^{2},
$$

where $\eta$ takes into account the deviation of the diffusion coefficient from its minimum value (in the nominal Bohm diffusion limit $\eta=1$ ). In the standard shock acceleration theory, the momentum index of accelerated electrons $\gamma_{\mathrm{s}}=4$, and the ratio of the upstream and downstream magnetic fields, $\kappa=1 / \sqrt{11}$.

In Fig. 1 the spectral points of G1.9+0.3 are compared with the theoretical predictions for synchrotron radiation in the upstream and downstream regions [6]. The calculations are performed 
for two values of the parameter $\eta$ characterising the acceleration efficiency: $\eta=1$ (Bohm diffusion regime) and 20 times slower $(\eta=20)$. The good (better than $20 \%$ ) agreement of the spectral points with the theoretical curves for $\eta=20$ tells us that in G1.9+0.3 electrons are accelerated only at the $5 \%$ efficiency level.

Although in the paper of [10] the spectral points are not explicitly presented, thus the direct comparison with our results is not possible, the conclusions based on our study of the X-ray spectrum of G1.9+0.3 seems to be in agreement with their results.
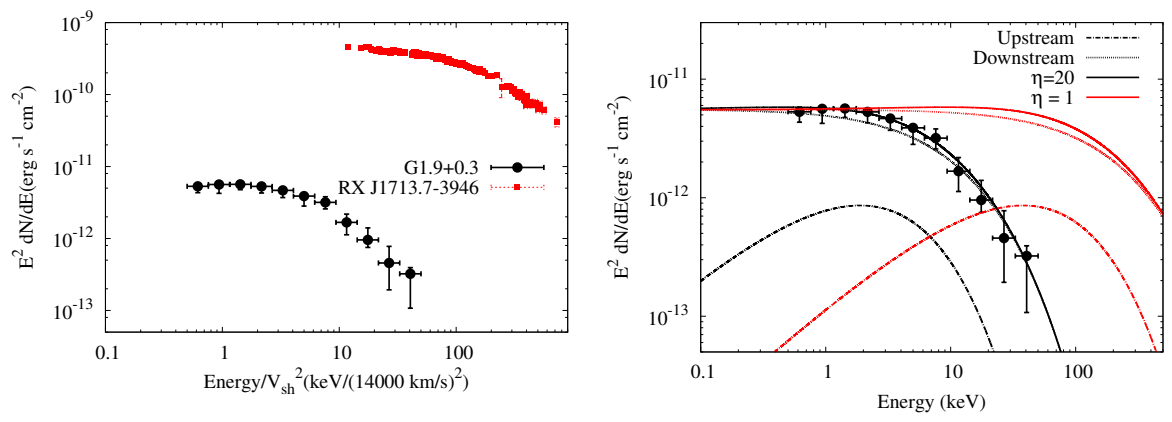

Figure 1: Left: The spectral points of G1.9+0.3 (this work; black circles) and RX J1713.4-3946 (red square) from [16]. The energies of the points of RX J1713.4-3946 are rescaled by the factor of the square of the ratio of shock speeds of J1713.4-3946 and G1.9+0.3: $(14,000 \mathrm{~km} / \mathrm{s} / 4000 \mathrm{~km} / \mathrm{s})^{2}=12.25$. Right: The spectral points of G1.9+0.3 (this work) compared to the predictions of synchrotron radiation of the shock accelerated electrons in the downstream and upstream regions [6]. The calculations correspond to two regimes of diffusion: (1) Bohm diffusion, $\eta=1$ and (2) 20 times faster diffusion, that is, $\eta=20$.

\begin{tabular}{l|l|l|l|l}
\hline Model & PL index & Cutoff $(\mathrm{keV})$ & $\beta$ & $\chi^{2} /$ d.o.f. \\
\hline \hline PL & $2.54(2.52-2.56)$ & & & $1089.4 / 666$ \\
\hline PL+ecut & $2.04(1.98-2.10)$ & $11.8(10.5-13.3)$ & & $697.7 / 665$ \\
\hline PL+ecut $(\beta=0.5)$ & $1.65(1.60-1.70)$ & $1.68(1.50-1.90)$ & 0.5 & $686.2 / 665$ \\
\hline PL+ecut $(\beta=0.33)$ & $1.18(1.10-1.26)$ & $0.07(0.06-0.08)$ & 0.33 & $687.5 / 665$ \\
\hline PL+ecut $(\beta$ free $)$ & $1.62(1.48-1.75)$ & $1.41(1.30-1.55)$ & $0.48(0.40-0.56)$ & $685.8 / 664$ \\
\hline
\end{tabular}

Table 1: Spectral fitting results for G1.9+0.3

\section{Relativistic electrons and magnetic fields}

The joint treatment of X-ray and $\gamma$-ray data, under the simplified assumption that the same electron population is responsible for the broad-band radiation through the synchrotron and inverse Compton channels, provides information about the magnetic field and the total energy budget in relativistic electrons. G1.9+0.3 has been observed in VHE $\gamma$-ray band with the H.E.S.S. Cherenkov telescope system. Although no positive signal has been detected [17], the $\gamma$-ray flux upper limits allow constraints on the the average magnetic field in the X-ray and $\gamma$-ray production region. A magnetic fields of more than $10 \mu \mathrm{G}$ was derived in [17]. Under certain assumptions, the magnetic field can be constrained also based only on the X-ray data. In the "standard" 
shock acceleration scenario, electrons are accelerated with the power-law index $\alpha=2$. However because of the short radiative cooling time, their spectrum of highest energy electrons (the $\mathrm{X}$-ray producers) becomes steeper, $\alpha=2 \rightarrow 3$. Consequently, in the downstream region, where the bulk of the synchrotron radiation is produced, $\mathrm{X}$-rays have a photon index $\Gamma=2$. The synchrotron cooling time can be expressed through the magnetic field and the X-ray photon energy: $t_{\text {synch }} \simeq 50(B / 100 \mu \mathrm{G})^{-3 / 2}(\varepsilon / 1 \mathrm{keV})^{-1 / 2}$ years. Thus for $\varepsilon \sim 1 \mathrm{keV}$ and the age of the SNR $\sim 150 \mathrm{yr}$, we find that the magnetic field should be larger than $50 \mu \mathrm{G}$.

The combined Chandra and NuSTAR data cover two decades in energy, from sub-keV to tens of keV. This allows derivation of the energy distribution of electrons, $W(E)=E^{2} \mathrm{~d} N_{\mathrm{e}} / \mathrm{d} E$ in the most interesting region around the cutoff. The results shown in Fig.2 are obtained using the Markov Chain Monte Carlo (MCMC) code Naima developed by V. Zabalza ${ }^{1}$. It is assumed that the magnetic field is homogeneous both in space and time. The results shown in Fig.2 are calculated for the fiducial value of the magnetic field $B=100 \mu \mathrm{G}$, however they can be rescaled for any other value of the field. We note that while the shape of the spectrum does not depend on the strength of the magnetic field, the energies of individual electrons scale as $E \propto B^{-1 / 2}$, and the total energy contained in electrons scales as $\propto B^{-2}$. Since in the "standard" diffusive shock acceleration (DSA) scenario the synchrotron X-ray flux is contributed mainly by the downstream region, the results in Fig. 2 are relevant to the energy distribution of electrons from the same region. For comparison, the energy distribution of electrons calculated using the formalism of [6] is shown.

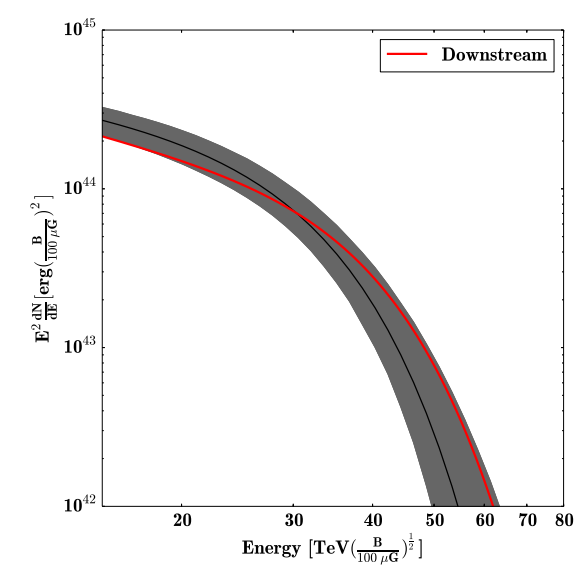

Figure 2: Electron spectrum from the X-ray data points (black curve and shaded area) and theoreticallly predicted integrated electron spectrum in a young SNR (red curve) assuming a fast diffusion with $\eta=20$. Also shown is the contribution from the downstream region.

\section{Conclusions}

SNRs are believed to be the major contributors to the Galactic CRs. The recent detections of $\mathrm{TeV}$ emission from more than ten young SNRs (of the age of a few thousand years or younger), demonstrates the ability of these objects to accelerate particles, electrons and/or protons, to energies

\footnotetext{
${ }^{1}$ https://github.com/zblz/naima
} 
up to $100 \mathrm{TeV}$. Yet, we do not have observational evidence of the extension of hard $\gamma$-ray spectra well beyond $10 \mathrm{TeV}$. Therefore one cannot claim an acceleration of protons and nuclei by SNRs to $\mathrm{PeV}$ energies. On the other hand, one cannot claim the opposite either, given the possibility that the acceleration of $\mathrm{PeV}$ protons and nuclei could happen at the early stages of evolution of SNRs when the shock speeds exceed $10,000 \mathrm{~km} / \mathrm{s}$. Indeed, the theoretical studies of recent years show that the best candidates for accelerators operating as PeVatrons are very young (less than 100 years old) supernova remnants in dense environments (see e.g. [18]). Then, the escape of the highest energy particles at later stages of evolution of SNRs can explain the spectral steepening of gamma-ray spectra at multi-TeV energies from $\geq 1$ thousand year old remnants.

In this regard, the youngest known SNR in our Galaxy, G1.9+0.3, with a measured shock speed of $14,000 \mathrm{~km} / \mathrm{s}$, seems to be a unique object in our Galaxy to explore the potential of SNRs for acceleration of protons and nuclei to PeV energies. Such measurements have been performed with the H.E.S.S. array of Cherenkov telescopes. Unfortunately, no positive signal has been detected. On the other hand, the recent observations of G1.9+0.3 in hard X-rays by NuSTAR provide unique information about the acceleration efficiency of electrons. Together with Chandra data at lower energies, these data allow model-independent conclusions. Although the general shape of the energy spectrum of X-rays is in very good agreement with predications of the diffusive shock-acceleration theory, the acceleration rate appears an order of magnitude slower relative to the maximum acceleration rate achieved in the nominal Bohm diffusion limit.

It should be noted that the deviation from the Bohm diffusion concerns not only the larger absolute value of the diffusion coefficient, but also the energy dependence of the latter. The diffusion coefficient written in the conventional form $D(E) \propto E^{\lambda}$, allows consideration of diffusion in a broad range of acceleration regimes. Then, in the synchrotron-loss-dominated regime, the energy distribution of electrons in the cutoff region has an exponential form, $N(E) \propto \exp \left[-\left(E / E_{0}\right)^{\beta_{\mathrm{e}}}\right]$ with $\beta_{\mathrm{e}}=\lambda+1$ (see Eq.(19) from [6]). Correspondingly, the index $\beta$ characterising the spectrum of synchrotron radiation in the cutoff region, is $\beta=(1+\lambda) /(3+\lambda)$. In particular, the values of $\lambda=0,1 / 3$, and 1 , which correspond to the energy-independent, Kolmogorov, and Bohm diffusion regimes, give $\beta=1 / 3,2 / 5$, and $1 / 2$, respectively. We note that the energy-independent diffusion in the synchrotron-loss-dominated regime results in an X-ray spectrum similar to the one formed in the case of the Bohm diffusion but in the age-limited regime. However, strictly speaking, because of the escape, the electrons in the age-limited regime of acceleration might have a steeper spectrum, that is, $\beta_{\mathrm{e}} \geq 1$. Although the results of the current analysis give a preference to the interval of $\beta=0.4-0.56$, the value of $1 / 3$ cannot be excluded (see Table 1 ).

To a certain extent, the relatively low acceleration rate of electrons in G1.9+0.3, in terms of the nominal rate of acceleration in the Bohm diffusion limit (for the given speed of the shock), is a surprise outcome, especially when compared with SNRs in which the acceleration of electrons proceeds in the regime close to the Bohm diffusion. For comparison, the detailed modelling of young SNRs Cas A [19] and RX J1713.4-3946 [15], shows that the $\eta$ parameter characterising the acceleration efficiency cannot significantly deviate from $\eta=1$. If the acceleration of protons and nuclei proceeds in the same manner as the electron acceleration, this result would imply inability of G1.9+0.3 to operate as a PeVatron. Apparently, the observations of G1.9+0.3 alone are not sufficient to decide whether or not this conclusion can be generalised for other SNRs. 


\section{References}

[1] Hillas, A. M., Evolution of ground-based gamma-ray astronomy from the early days to the Cherenkov Telescope Arrays, APh 43 (2013) 19

[2] Blasi, P., The origin of galactic cosmic rays, A\&ARv 21 (2013) 70

[3] Aharonian, F. A., Gamma rays from supernova remnants, APh 43 (2013) 71

[4] Malkov, M. A., Drury, L. O., Nonlinear theory of diffusive acceleration of particles by shock waves, RPPh 64 (2001) 429

[5] Aharonian, F. A., Atoyan, A. M., On the origin of TeV radiation of SN 1006, A\&A 351 (1999) 330

[6] Zirakashvili, V. N., Aharonian, F., Analytical solutions for energy spectra of electrons accelerated by nonrelativistic shock-waves in shell type supernova remnants, A\&A 465 (2007) 695

[7] Reynolds, S. P., et al., The Youngest Galactic Supernova Remnant: G1.9+0.3, ApJ 680 (2008) L41

[8] Green, D. A., et al., The radio expansion and brightening of the very young supernova remnant G1.9+0.3, MNRAS 387 (2008) L54

[9] Reynolds, S. P., et al., X-Ray Spectral Variations in the Youngest Galactic Supernova Remnant G1.9+0.3, ApJ 695 (2009) L149

[10] Zoglauer, A., et al., The Hard X-Ray View of the Young Supernova Remnant G1.9+0.3, ApJ 798 (2015) 98

[11] Reynolds, S. P., Supernova Remnants at High Energy, ARA\&A 46 (2008) 89

[12] Wik, D. R., et al., NuSTAR Observations of the Bullet Cluster: Constraints on Inverse Compton Emission, ApJ 792 (2014) 48

[13] Borkowski, K. J., et al., Radioactive Scandium in the Youngest Galactic Supernova Remnant G1.9+0.3, ApJ 724 (2010) L161

[14] Uchiyama, Y., et al., Extremely fast acceleration of cosmic rays in a supernova remnant, Natur 449 (2007) 576

[15] Zirakashvili, V. N., Aharonian, F. A., Nonthermal Radiation of Young Supernova Remnants: The Case of $R X$ J1713.7-3946, ApJ 708 (2010) 965

[16] Tanaka, T., et al., Study of Nonthermal Emission from SNR RX J1713.7-3946 with Suzaku, ApJ 685 (2008) 988-1004

[17] H.E.S.S. Collaboration, et al., TeV $\gamma$-ray observations of the young synchrotron-dominated SNRs G1.9+0.3 and G330.2+1.0 with H.E.S.S., MNRAS 441 (2014) 790

[18] Schure, K. M., Bell, A. R., Cosmic ray acceleration in young supernova remnants, MNRAS 435 (2013) 1174

[19] Zirakashvili, V. N., et al., Nonthermal Radiation of Young Supernova Remnants: The Case of CAS A, ApJ 785 (2014) 130

[20] Fritz, K. D., Synchrotron emission spectra from shockwaves in active galactic nuclei - an energy and space dependent diffusion coefficient, A\&A 214 (1989) 14 